\title{
UNESCO Core Curriculum and Teaching Resources
}

\author{
Darryl Macer PhD \\ Regional Advisor, Regional Unit for Social and Human Science in Asia and the Pacific, \\ Bangkok, Thailand \\ Email: d.macer@unesco.org
}

The task of a teacher includes empowering their student to develop their maturity as individuals as well as being able to cooperative members of changing societies. Student need to be prepared so they are able to apply knowledge to make good decisions during their life. Basic to fulfilling many tasks, teachers themselves need to be in the right situation. How can we train teachers and sustain their motivation to take upon this sacred vocation? How can create communities that tolerate these goals?

This paper will look at the roles and challenges for teachers and institutions, with some comparisons between countries and trends. There will be examples of some training strategies and methods being used in the multidisciplinary area of bioethics. Bioethics is looking at ethical issue relating to life science and technology, or could even be called the love of life. Ethics is a concept balancing benefits and risks of choice and decisions. The underlying heritage of ethics can be seen in all cultures, religions, and in ancient writings around the world. The turbulent times of today have challenged some of the traditional structures in the relationships between human beings within their society, with nature and God. How can we empower teachers to make a special contribution in the wider context of constructing a mature society. Mature means a person, or a society that can balance the benefits and risk of alternative options, and make well considered decisions, and talk about it.

There are at least four strategies that thinking person need to cope with the turbulent times.

1. Descriptive ethics to enable people to accurately assess the situation. People need to understand the way they and other people view life, their moral interactions and responsibilities with other in their life.

2. Prescriptive ethics is to tell others what is ethically good and bad, or what principles are the most important in making such decisions. It may also be to say something or someone

has rights, and others have duties to them. It is a basic for sound policy making, and law, and empowering people to make decision in their life.

3. Interactive ethics is decision and debate between people, groups within society, and communities. Such dialogue skills are necessary to live harmoniously with others. The tensions in turbulent times are even more stretched yet we must continue to teach people to get along, and empower teachers to interact across perceived boundaries of discipline, culture, religion and ideology.

4. Practicality is essential if teachers are expected to continue teaching, and students will continue their interest in the matter.

This paper will gives some examples of teaching critical thinking through both environmental and medical ethics issues. Critical thinking is essential for empowering person to cope with changing times. How do we promote the creation of ideas, and individuality in an era of 
globalization? The rapid progress of technology has led to challenges in the way that we live. The systems and the patterns that are seen in the relationships between patients, families, health professionals, and the society in general changed. Appropriate education requires teachers to apply methods and resources appropriately and to develop the decision making ability in students, teachers and policy makers so our society can evolve with the demands of the times.

The UNESCO Bioethics Core Curriculum sets out to introduce the bioethical principles of the Universal Declaration on Bioethics and Human Rights to university students. Bioethics teaching has not introduced in many universities in many countries. The UNESCO Core Curriculum can provide an incentive to start introducing such teaching. Its contents are based on the principles adopted in UNESCO. It therefore does not impose model or specific view of bioethics, but articulate ethical principles that are shared by scientific experts, policymakers and health professionals from various countries with different cultural, historical and religious backgrounds.

However, the core curriculum should not be treated as a comprehensive curriculum in bioethics. It is recognized that the content of the core curriculum does not necessarily cover all aspects of bioethics. Traditional issues that have not been included could be incorporated as examples that are pertinent to one or several of the Declaration's principles within the curriculum's framework.

We are working with institutions that have varying levels of experience with bioethics education, some of them at the early stage to introduce new bioethics courses, while others, are reviewing their curricula with a view to enriching it from cultural perspectives, taking into account the UNESCO Bioethics Core Curriculum. Also we are gathering feedback from bioethics education efforts, and examining existing bioethics curriculum developed at national and international level, so to decide either to follow transdisciplinary curricula across medical, environmental and science ethics or independent curricula specific for each discipline. 